\title{
The Effect of a Step Increase in Depth and Decay upon Dispersion of Coastal Effluent Discharges
}

\author{
Abdullrahman A. Al-Muqbali, Anton Purnama \\ Department of Mathematics, Sultan Qaboos University, Muscat, Oman \\ Email:s91616@student.squ.edu.om, antonp@squ.edu.om
}

How to cite this paper: Al-Muqbali, A.A. and Purnama, A. (2022) The Effect of a Step Increase in Depth and Decay upon Dispersion of Coastal Effluent Discharges. Applied Mathematics, 13, 37-55.

https://doi.org/10.4236/am.2022.131004

Received: December 12, 2021

Accepted: January 18, 2022

Published: January 21, 2022

Copyright $\odot 2022$ by author(s) and Scientific Research Publishing Inc. This work is licensed under the Creative Commons Attribution International License (CC BY 4.0).

http://creativecommons.org/licenses/by/4.0/

\begin{abstract}
Coastal wastewater-discharged effluents contain a mixture of pollutants with decay rates that vary with water depth. Analytical models using a two-dimensional advection-diffusion equation are presented to study the effects of a cross-stream sudden depth change and decay on mixing and dispersing steady discharge of effluents through a sea outfall. The solutions are illustrated graphically by plotting contours of concentration, resembling snapshots of discharged effluent plumes in the far-field. Different shapes of effluent plumes are observed due to the variability of length of the step seabed, and the concentration at the step seabed is formulated to measure how much has discharged effluents dispersed into or out of the shallow coastal waters.
\end{abstract}

\section{Keywords}

Advection-Diffusion Equation, Flat Seabed, Method of Image, Step Seabed, Variability of Decay

\section{Introduction}

Steady discharge of wastewater effluents through marine outfall systems into the sea includes (treated) municipal wastewaters [1] [2] [3], cooling waters [4], and brine effluents [5] [6]. Occasionally, due to the desalination plant maintenance, discharged brine effluents may consist of corrosion products, toxic antifoulants and antiscalants [7] [8]. These types of effluents are subject to (temporal) decay and may contain some unknown (emerging) chemicals, where some of the components are not yet identified and their toxicity cannot be explained. Decay mechanisms [9] [10] include consumption by bacteria or radioactive decay (decay 
uniform across the flow), heat loss or evaporation through the surface (decay decreasing with depth), and break up or dissolve by turbulence (decay proportional to the flow). For calm sea conditions, the time scales for transverse mixing can be of order a day and thus comparable with the time scales for effluents decay. So, the effect of decay cannot be regarded as a minor perturbation that simply lowers the discharged effluent concentration.

One factor affecting the dilution and spreading of wastewater effluent discharge in coastal waters is the seabed depth profiles [11] [12] [13] [14] [15], which are typically ranging between a sloping sandy beach and a mountainous coast with rock sea cliffs, where water depth gets very deep within a short distance from the coastline. If the discharge of wastewater effluents into the sea cannot be avoided, then it should be done as optimally as possible to ensure that the environmental impact on coastal waters is minimized. Coastal regions and beaches are important for fisheries, local recreation and tourism and for conservation areas.

Modeling studies of the effects of a step increase in depth and decay that decreases or increases with water depth in dispersing steady coastal discharged effluents from a sea outfall in the far-field is investigated using a two-dimensional advection-diffusion equation with a point source (see for example [16] [17]). The use of analytical solutions has been a key strategy for the basis of engineering design of marine outfall systems and for assessing the potential impacts. In terms of the practical applicability, it is well recognized that the mathematical model can be applied as benchmark testing to perform preliminary worst-case assessments [16] [17] [18]. If this easy-to-use assessment indicates no impacts at all, no further action is needed and the use of more sophisticated and timeconsuming three-dimensional hydrodynamic and water quality modeling can be avoided.

\section{A Simple Flat Seabed Model}

Some seabed depth profiles are extremely flat such that the variations in water depth become insignificant. Therefore, as a reference, we introduce first a highly simplified model of the flat seabed with a constant depth, and for simplicity, the other complexities, such as tidal motions, density and temperature, are ignored. The shoreline, which in this case, is a continuation of the rocky sea cliffs, is assumed to be straight, and the effluent is discharged at a steady rate $Q$ from a point at $\left(x=0, y=\alpha h_{0}\right)$, where $h=h_{0}$ is an arbitrary reference depth. The (drift) longshore current with speed $U_{0}$ is assumed to be in the $x$-direction at all times. The decay rate $\mu_{0}$ with a typical value up to 0.5 day $^{-1}$ is used to represent decay of faecal in recreational coastal waters [19], decay of dissolved oil (biological consumption of hydrocarbons) [20], and decay of biological oxygen demand [21]. The dispersion processes are represented by the coefficient of dispersivity $D_{0}$, and dispersion in the $x$-direction is neglected, as the discharged effluent plumes in steady currents become very elongated in the flow direction. 
The marine outfall systems are commonly installed with multiport diffusers designed to rapidly mix and dilute the discharged effluents with the receiving sea currents, and thus, it is also assumed that discharged effluent concentration is vertically well-mixed over the water depth.

The two-dimensional advection-diffusion equation for the concentration $c(x, y)$, incorporating a first-order decay for the non-conservative discharged effluent, is given by

$$
h_{0} \mu_{0} c+h_{0} U_{0} \frac{\partial c}{\partial x}-h_{0} D_{0} \frac{\partial^{2} c}{\partial y^{2}}=Q \delta(x)\left[\delta\left(y-\alpha h_{0}\right)+\delta\left(y+\alpha h_{0}\right)\right],
$$

where the Dirac delta function $\delta(*)$ is used to represent the position of a source at $\left(x=0, y=\alpha h_{0}\right)$, and an imaginary source at $\left(x=0, y=-\alpha h_{0}\right)$ has been added to satisfy the no-slip boundary condition at $y=0$. Note that, the first decay term can be eliminated from Equation (1) by rewriting $c=c_{*} \exp \left(-\mu_{0} x / U_{0}\right)$.

For the graphical representation of solutions, we define dimensionless quantities

$$
y=Y h_{0}, \quad x=X h_{0} \text { and } C_{*}(x, y)=C_{*}(X, Y) Q / U_{0} h_{0}^{2},
$$

Equation (1) reduces to

$$
\frac{\partial C_{*}}{\partial X}-\frac{1}{\lambda} \frac{\partial^{2} C_{*}}{\partial Y^{2}}=\delta(X)[\delta(Y-\alpha)+\delta(Y+\alpha)]
$$

and the solution for $X \geq 0$ is given by

$$
\begin{aligned}
C & =C_{*} \exp (-\gamma X) \\
& =\sqrt{\frac{\lambda}{4 \pi X}}\left[\exp \left\{-\frac{\lambda(Y-\alpha)^{2}}{4 X}\right\}+\exp \left\{-\frac{\lambda(Y+\alpha)^{2}}{4 X}\right\}\right] \exp (-\gamma X)^{\prime}
\end{aligned}
$$

where the parameter $\lambda=U_{0} h_{0} / D_{0}$ represents discharged effluent plumes elongation in the $x$-direction, and $\gamma=\mu_{0} h_{0} / U_{0}$ represents the loss rate of discharged effluents.

To investigate the uncertainty and variability of sea conditions in these parameters, Figure 1 shows the possible values of $\lambda$ and $\gamma$ for some relevant measured values of $U_{0}$ and $D_{0}$ in the shallow coastal waters with depth $h_{0}=5$ and 10. It is expected that the larger the values of $\lambda$, the more elongated the effluent plumes, which are mostly due to a stronger current $U_{0}$ with less transverse dispersivity $D_{0}$. Similarly, bigger values of $\gamma$ are mostly due to a higher rate of decay $\mu_{0}$ during a calmer sea condition. We note that, since the value of $\gamma$ is naturally small, in order for the effect of decay to be noticeable, the values of $\gamma$ should be sufficiently large. From Figure 1, the reference values of $\lambda=0.15$ and $\gamma=0.0003$ will be used in the subsequent plots, unless stated otherwise.

The contours of concentration Equation (2) are plotted in Figure 2 for discharging effluents from two positions of a point source at $\alpha=20$ and $\alpha=80$, which corresponds to a sea outfall length in the range of $100-200 \mathrm{~m}$ and $400-$ 

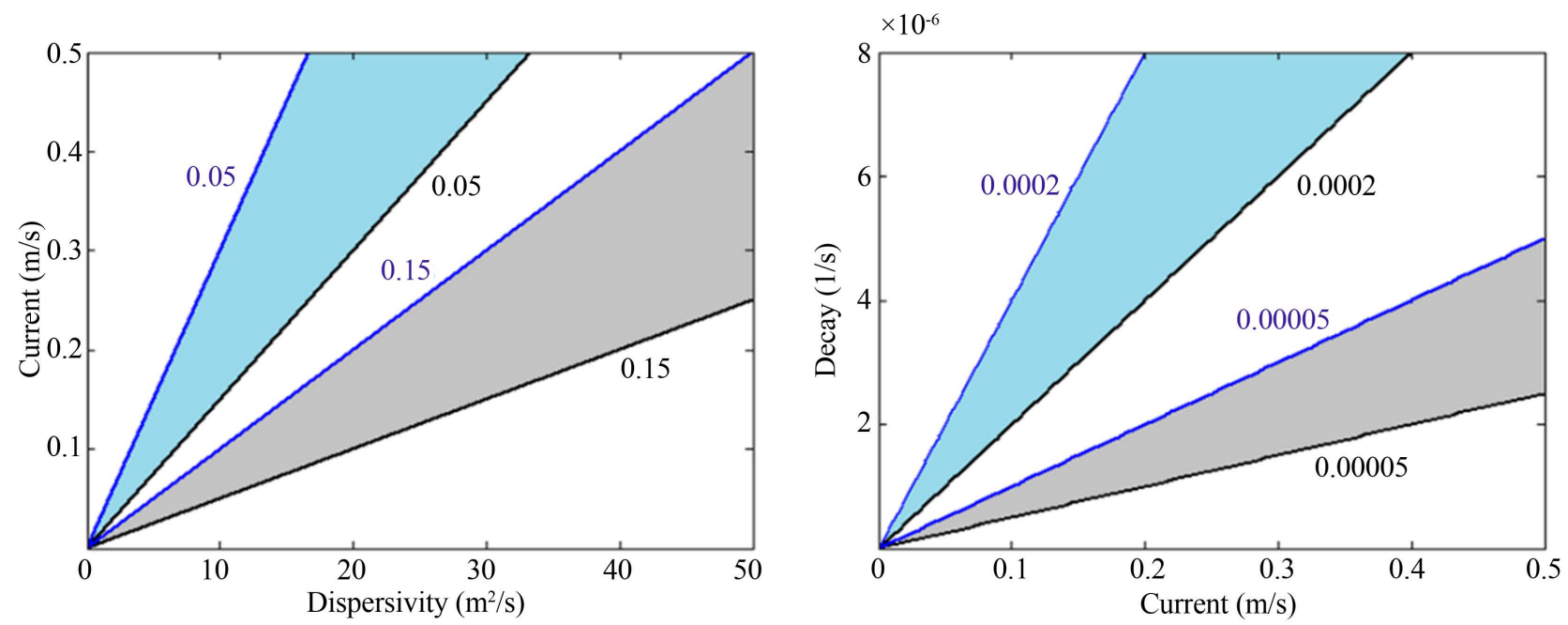

Figure 1. The parameters $\lambda$ (left) and $\gamma \quad$ (right) for $h_{0}=5 \quad$ (blue) and $h_{0}=10$ (black).
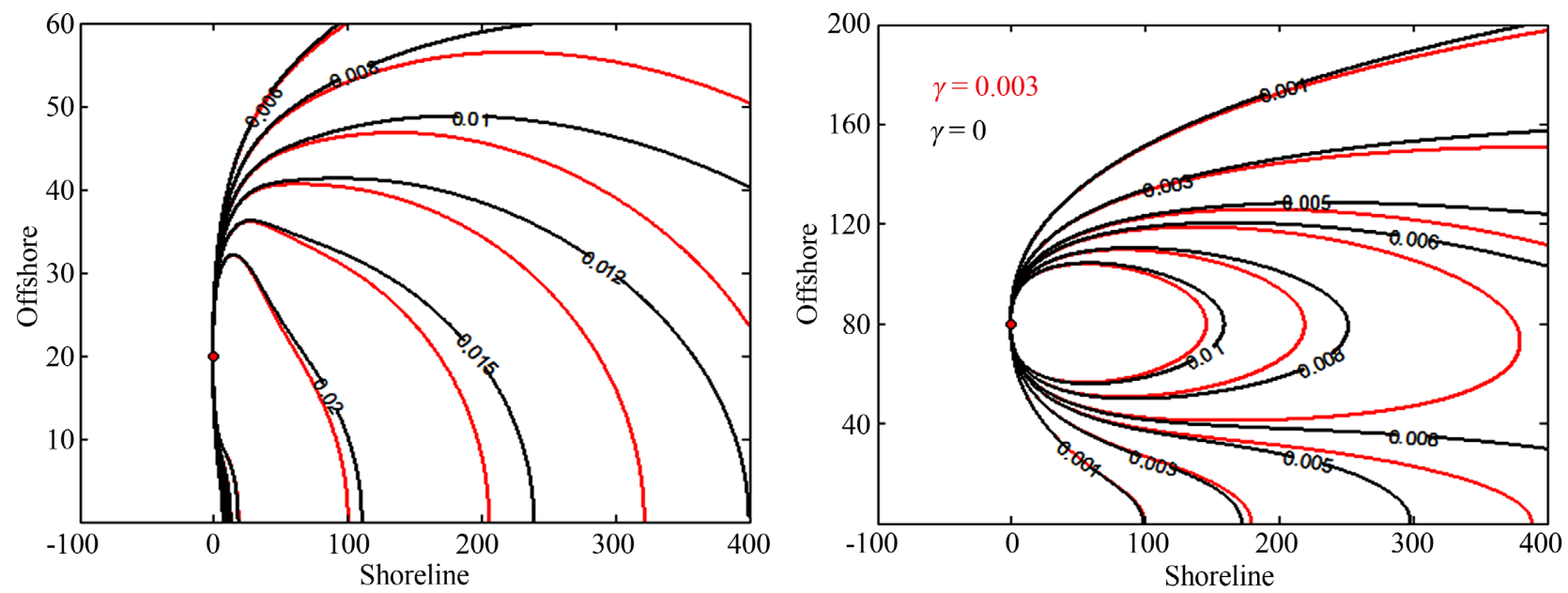

Figure 2. Contours of concentration for steady effluent discharge on the flat seabed from a point source $\alpha=20$ (left) and $\alpha=80$ (right).

$800 \mathrm{~m}$ respectively for $h_{0}$ in the range of $5-10 \mathrm{~m}$. We observed that, due to loss of discharged effluents, the contours $(\gamma>0)$ are smaller than that of no decay $\gamma=0$, plotted in black. As shown in the right column when $\lambda=0.2$, the discharged effluent plumes are more elongated (in the flow direction).

We observed that from Figure 2 (right), it appears that the discharged effluent plumes from a source at $\alpha=80$ almost symmetry about the centerline as if they do not feel the presence of shoreline. Eventually it bends over towards and reaches the shoreline at a far downstream distance $X>300$. However, from Figure 2 (left), for the effluent discharge near the shoreline at $\alpha=20$, due to the no-slip condition, the effluent plumes attaching to the shoreline; and the effect of transverse dispersivity appears to be more dominant compared to that of the advection current. Thus, a higher build-up to unacceptable concentration levels will occur close to the shoreline. Therefore, the appropriate measure for assessing the environmental impact of discharging effluents into the sea would 
be the maximum concentration at the shoreline [5] [12] [13]. A typical standard regulatory criterion would state does not exceed a certain prescribed safety level of concentration anywhere along the shoreline to control public health risks in some areas where coastal waters are used for swimming and recreational purposes.

A plot of effluent flux can be used to illustrate how a discharged effluent plume is dispersing and spreading out from a point source by drawing an arrow at each grid point $(x, y)$, where the direction of the arrow indicates the direction of motion of the discharged effluent, and the length of the arrow is proportional to the magnitude of the flux at that point. Since longitudinal dispersion has been neglected, the flux in the $x$-direction is due to advection, $\left(Q / h_{0}\right) F_{x}=h_{0} U_{0} c$; and the flux in the $y$-direction is due to dispersion, $\left(Q / h_{0}\right) F_{y}=-h_{0} D_{0} \partial c / \partial y$. From Equation (2), the dimensionless form of the effluent flux are

$$
F_{x}=\sqrt{\frac{\lambda}{4 \pi X}}\left[\exp \left(-\frac{\lambda(Y-\alpha)^{2}}{4 X}\right)+\exp \left(-\frac{\lambda(Y+\alpha)^{2}}{4 X}\right)\right] \exp (-\gamma X)
$$

and

$$
\begin{aligned}
F_{y}= & \sqrt{\frac{\lambda}{4 \pi X}}\left[\frac{(Y-\alpha)}{2 X} \exp \left(-\frac{\lambda(Y-\alpha)^{2}}{4 X}\right)\right. \\
& \left.+\frac{(Y+\alpha)}{2 X} \exp \left(-\frac{\lambda(Y+\alpha)^{2}}{4 X}\right)\right] \exp (-\gamma X) .
\end{aligned}
$$

Due to diffusion process, Figure 3 shows the motion of a discharged effluent plume on the flat seabed as it spreads from the regions of high concentration. For a point close to the shoreline at $\alpha=10$ (Figure 3 left), we observe that in the offshore region $Y \geq \alpha$, the effluent plume is tending to move further away from the shoreline. However, in the nearshore region $0 \leq Y<\alpha$, the effluent
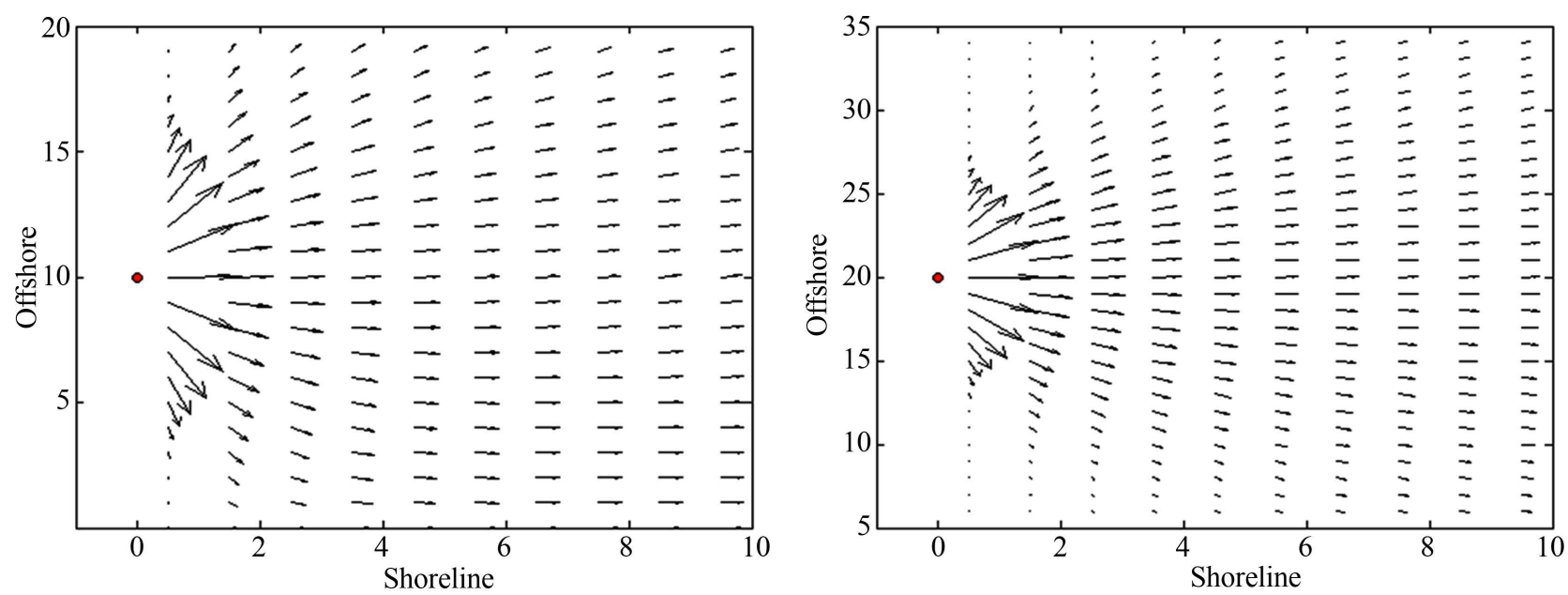

Figure 3. Flux of concentration for discharged effluents on the flat seabed from a point source at $\alpha=10$ (left) and $\alpha=20$ (right) for the case of no decay $\gamma=0$. 
plume spreads towards the shoreline, and reaches the shoreline at a relatively short downstream distance, then due to the no-slip condition, the plume continues to move in direction of the flow. By moving the point source further away from the shoreline at $\alpha=20$ (Figure 3 right), the effluent plume is advected by the flow before it reaches the shoreline at a far downstream distance.

Substituting $Y=0$ in Equation (2), the concentration at the shoreline

$$
C(X, 0)=\sqrt{\frac{\lambda}{\pi X}} \exp \left(-\frac{\lambda \alpha^{2}}{4 X}-\gamma X\right) .
$$

As shown in Figure 4, Equation (3) for discharging effluents from a point source at $\alpha=20$ and $\alpha=80$ on a flat seabed has a maximum value. The long tails of the graphs for discharges at $\alpha=20$ are the results of the induced effects of the no-slip condition at $y=0 \quad$ (see Figure 3).

By differentiating, it is straightforward to obtain the maximum value

$$
C_{\max }=\sqrt{\frac{1+\sqrt{1+4 \lambda \gamma \alpha^{2}}}{\pi \alpha^{2}}} \exp \left(-\frac{\sqrt{1+4 \lambda \gamma \alpha^{2}}}{2}\right),
$$

which occurs at $X_{\max }=\lambda \alpha^{2} /\left(1+\sqrt{1+4 \lambda \gamma \alpha^{2}}\right)$. For no decay $\gamma=0$, $C_{\max }=\sqrt{2 / \pi \mathrm{e} \alpha^{2}}$ and $X_{\max }=\lambda \alpha^{2} / 2$. This suggests that, since $C_{\max }$ is inversely proportional to $\alpha$, the concentration build-up close to the shoreline can be avoided by building a long sea outfall. The maximum concentration is about 0.0242 for a short point source $\alpha=20$, and for large values of $\alpha$, it decreases to 0.006 for a long point source $\alpha=80$. Similarly, due to loss of discharged effluents at a slightly higher rate of $\gamma=0.0003, C_{\max }$ decreases slightly to 0.024 for $\alpha=20$; and decreases significantly to 0.0053 for $\alpha=80$.

For a long point source at $\alpha=80$, due to loss of discharged effluents, the position of the maximum concentration is smaller than that of no decay $\gamma=0$. As the decay rate $\gamma$ increases, $X_{\max }$ decreases from 480 for $\gamma=0$ to about 389
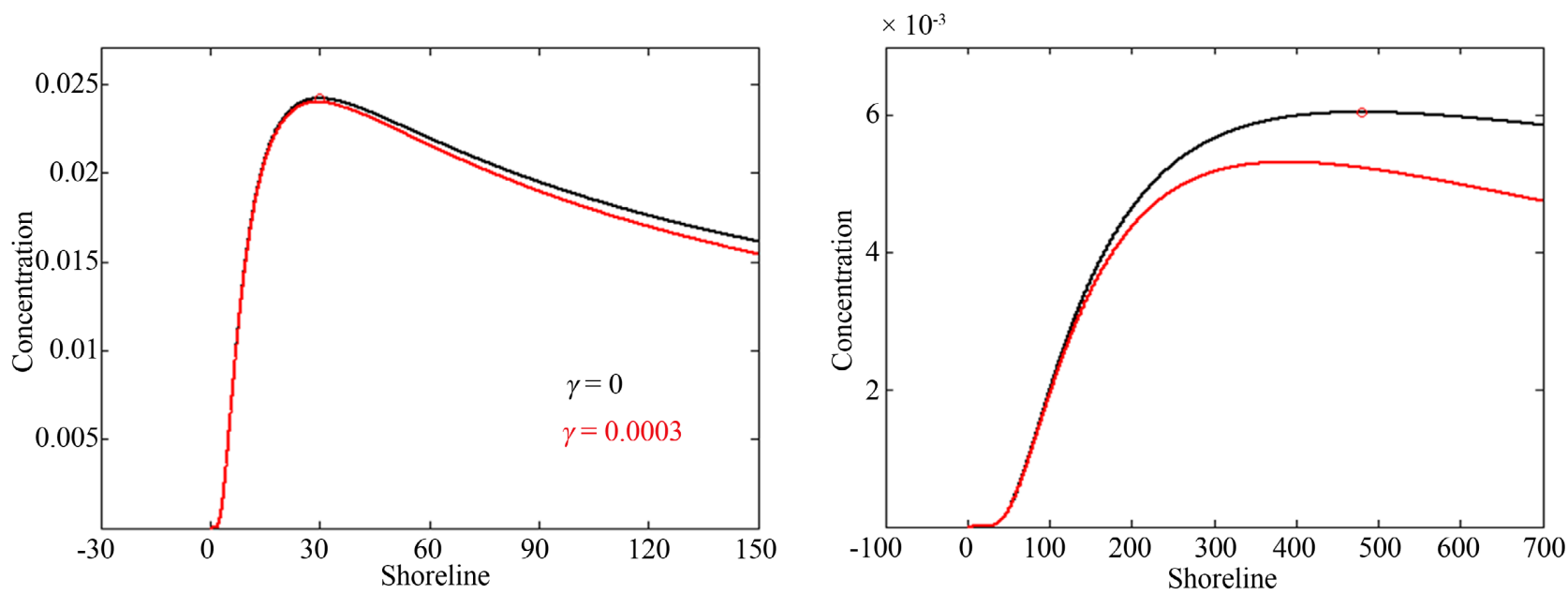

Figure 4. Concentration at the shoreline for steady effluent discharge on the flat seabed from a point source at $\alpha=20$ (left) and $\alpha=80$ (right). 
for $\gamma=0.0003$. Thus, we concluded that the induced effect of the no-slip condition at the shoreline $y=0$ can be ignored for discharging effluents from a long point source at $\alpha=80$.

\section{A Step Seabed Model}

The sandbags landfill is one of the popular methods to restore and protect the beach erosion from constant wave attack. Due to the pillow shape of sandbags, the beach (face) makeover of piling sandbags creates a profile of steps seabed along the shoreline. In the oceanography textbooks, going further seaward from the shore, the first submerged region is termed continental shelf. The seaward limits of the shelf are determined by the distinct change in depths between the shelf and its adjacent continental slope. Thus, a seabed depth profile is typically depicted as a shallow depth flat seabed coming in contact with a deeper one. As a first attempt to study the effect of variations in water depth, we consider, as shown in Figure 5 (left), a step seabed depth profile

$$
h(y)=\left\{\begin{array}{cc}
h_{0}, & 0 \leq y<\ell h_{0} \\
h_{1}, & y>\ell h_{0}
\end{array},\right.
$$

where the sudden cross-stream water depth change occurs at a discontinuity line $y=\ell h_{0} \quad(\ell>0)$ and $r=h_{1} / h_{0}>1$ is the ratio of water depths $\left(h_{1}>h_{0}\right)$. Note that if $r=1$ (and $\ell=0$ ), there is no depth change, and a seabed depth profile is exactly that of a simple flat seabed of depth $h=h_{0}$ (and $h=h_{1}$ ).

For a turbulent shallow-water flow over a smooth bed, the variations in the $y$-direction of current $U_{1}$ and dispersivity $D_{1}$ are assumed as the power functions only of water depth $h$ [22], and for model application, we take $U_{1}$ to be proportional to $h^{1 / 2}$ and $D_{1}$ to $h^{3 / 2}$ [11] [12]. That is, $U_{1}=U_{0} r^{1 / 2}$ and $D_{1}=D_{0} r^{3 / 2}$. Also, we assume that the decay rate $\mu_{1}$ as a function of depth and proportional to $h^{\sigma+1 / 2}$ [9] [10], and thus, $\mu_{1}=\mu_{0} r^{\sigma+1 / 2}$. The effects of loss of

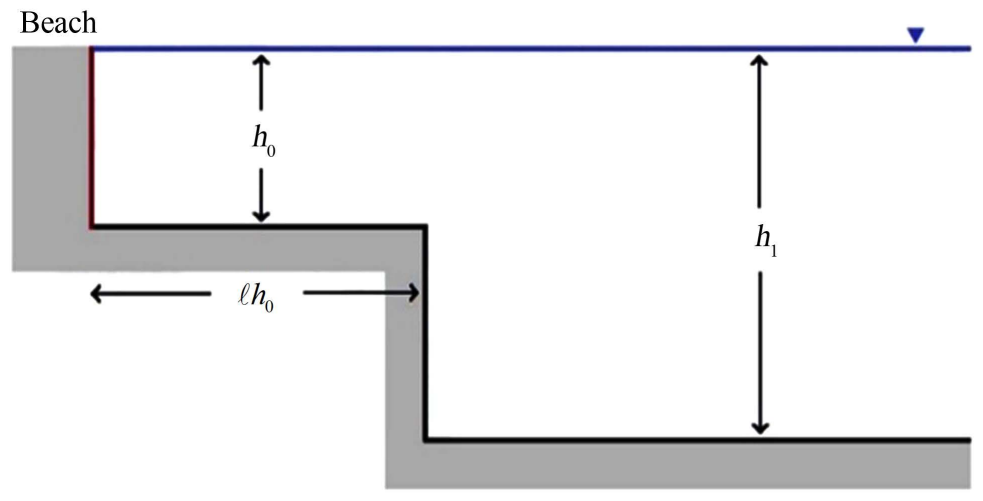

Seabed

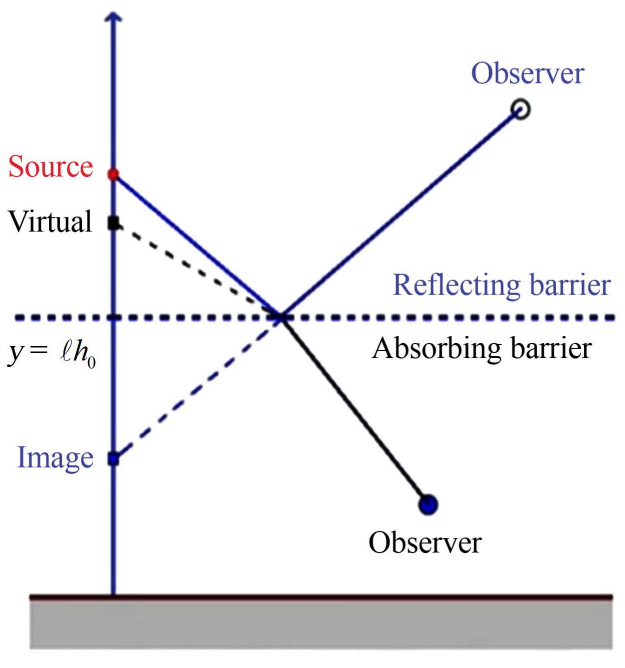

Shoreline

Figure 5. Cross-section view of the step seabed (left); and plan view of the method of image (right). 
discharged effluents that varies with depth can be illustrated according to the values of $\sigma$.

If $\sigma=-1 / 2$, then $\mu_{1}=\mu_{0}$, and the decay rate is independent of water depth. Radioactive decay or consumption by bacteria (at a rate unaffected by sunlight or turbidity) is examples of constant temporal decay. For $\sigma<-1 / 2$, the decay rate $\mu_{1}$ decreases with depth. For example, if $\sigma=-1$ then $\mu_{1}=\mu_{0} r^{-1 / 2}$ represents the decay rate that includes consumption by bacteria which are killed by sunlight at the surface, and air-water exchange (evaporation) at the surface [4] [9] [10]. For $\sigma>-1 / 2, \mu_{1}$ increases with depth, and if $\sigma=0$ then $\mu_{1}=\mu_{0} r^{1 / 2}$ represents the decay rate for the dissolution of oils or break up of clay flocs, which occurs most rapidly in the regions of the flow where the turbulence is energetic. In the subsequent plots, the reference values $r=2$ and $\sigma=-1 / 2$ will be used unless stated otherwise.

On writing the concentration, in dimensionless form, as

$$
C(X, Y)=\left\{\begin{array}{l}
C_{0}=C_{0^{*}} \exp (-\gamma X), \quad 0 \leq Y<\ell \\
C_{1}=C_{1^{*}} \exp \left(-\gamma r^{\sigma} X\right), \quad Y>\ell
\end{array},\right.
$$

the solution of a two-dimensional advection-diffusion equation for discharging effluents from a point source at $(X=0, Y=\alpha)$ on the step seabed can be obtained using the method of image [11] [12] [14] [15]. For example, as illustrated in Figure 4 (right, where the solid lines are considered as rays [11]), if a point source is in the offshore deeper region, then the concentration $C_{1}(X, Y)$ is due to the point source plus an imaginary point source on the other side of the reflecting barrier at $Y=\ell$. The concentration $C_{0}(X, Y)$ is due to a virtual source diffusing over the absorbing barrier at $Y=\ell$.

However, as there can be no discontinuities in either the concentration or its gradient across the line $Y=\ell$, the additional matching conditions are

$$
C_{0}=C_{1} \text { and } \frac{\partial C_{0}}{\partial Y}=r^{5 / 2} \frac{\partial C_{1}}{\partial Y} .
$$

The discontinuity line serves as a "gate" for discharging effluent plumes to cross over and spread into or out of the shallow nearshore region. Thus, to separate the effect of a sudden depth change (at the line $Y=\ell$ ) to that of the no-slip condition at the shoreline (at $Y=0$ ), we consider two values of $\ell$ : a short step seabed with $\ell \leq 15$ (to represent a sandbags landfill depth profile), where the presence of shoreline induces the effect of the no-slip condition in dispersing effluent discharged plumes; and a long step seabed with $\ell \geq 70$ (to represent a continental shelf depth profile), where a discharged effluent plume most likely does not feel the presence of shoreline.

\subsection{Short Step Seabed}

Older sea outfalls are typically short and some discharges are made through open channel at the shoreline, where higher build-up of effluents is expected in coastal waters close to and along the shoreline. For a relatively short step seabed 
with $\ell \leq 15$, we consider a source at $(X=0, Y=\alpha)$ where $\alpha>\ell$, that is sufficiently close to the discontinuity line $Y=\ell$. The concentration in the shallow region $C_{0}(X, Y)=C_{0^{*}} \exp (-\gamma X)$ is obtained due to a virtual source at $\left(X=0, Y=\beta_{1}\right)$ discharging at a rate $b_{1}$, and satisfies the no-slip boundary condition at $Y=0$. Thus, in dimensionless form, the advection-diffusion equation for $C_{0^{*}}(X, Y)$ is given by

$$
\frac{\partial C_{0^{*}}}{\partial X}-\frac{1}{\lambda} \frac{\partial^{2} C_{0^{*}}}{\partial Y^{2}}=b_{1} \delta(X)\left[\delta\left(Y-\beta_{1}\right)+\delta\left(Y+\beta_{1}\right)\right]
$$

and the solution for $X \geq 0$ is

$$
C_{0}=b_{1} \sqrt{\frac{\lambda}{4 \pi X}}\left[\exp \left\{-\frac{\lambda\left(Y-\beta_{1}\right)^{2}}{4 X}\right\}+\exp \left\{-\frac{\lambda\left(Y+\beta_{1}\right)^{2}}{4 X}\right\}\right] \exp (-\gamma X) .
$$

The concentration in the deeper region $C_{1}(X, Y)=C_{1^{*}} \exp \left(-\gamma r^{\sigma} X\right)$ is obtained due to a point source at $(X=0, Y=\alpha)$ and an imaginary source at $(X=0, Y=2 \ell-\alpha)$ discharging at a different rate $a_{1}$. Thus, the advectiondiffusion equation for $C_{1^{*}}(X, Y)$ is given by

$$
\frac{\partial C_{1^{*}}}{\partial X}-\frac{r}{\lambda} \frac{\partial^{2} C_{1^{*}}}{\partial Y^{2}}=\frac{1}{r^{3 / 2}} \delta(Y)\left[\delta(Y-\alpha)+a_{1} \delta(Y-2 \ell+\alpha)\right],
$$

and the solution for $X \geq 0$ is

$$
\begin{aligned}
C_{1}= & \frac{1}{2 r^{2}} \sqrt{\frac{\lambda}{\pi X}}\left[\exp \left\{-\frac{\lambda(Y-\alpha)^{2}}{4 r X}\right\}\right. \\
& \left.+a_{1} \exp \left\{-\frac{\lambda(Y-2 \ell+\alpha)^{2}}{4 r X}\right\}\right] \exp \left(-\gamma r^{\sigma} X\right) .
\end{aligned}
$$

The matching conditions at $Y=\ell$ are required for calculating $a_{1}, b_{1}$ and $\beta_{1}$, and thus, we obtain

$$
\begin{aligned}
& {\left[r^{2}+1+\left\{r^{2}+\frac{\beta_{1}+\ell}{\beta_{1}-\ell}\right\} \exp \left(-\frac{\lambda \ell \beta_{1}}{X}\right)\right] a_{1}} \\
& =\left[r^{2}-1+\left\{r^{2}-\frac{\beta_{1}+\ell}{\beta_{1}-\ell}\right\} \exp \left(-\frac{\lambda \ell \beta_{1}}{X}\right)\right]
\end{aligned}
$$

and

$$
b_{1} \exp (-\gamma X)\left[r^{2}+1+\left\{r^{2}+\frac{\beta_{1}+\ell}{\beta_{1}-\ell}\right\} \exp \left(-\frac{\lambda \ell \beta_{1}}{X}\right)\right]=2 \exp \left(-\gamma r^{\sigma} X\right) .
$$

We noted numerically that for $X<5.6, \exp \left(-\lambda \ell \beta_{1} / X\right)<0.001$, and thus,

$$
a_{1}=\frac{r^{2}-1}{r^{2}+1} \text { and } b_{1} \exp (-\gamma X)=\frac{2}{r^{2}+1} \exp \left(-\gamma r^{\sigma} X\right) \text {. }
$$

Further, for no decay $\gamma=0$ (and $\sigma=0), a_{1}+b_{1}=1$. From the previous section, for $r>1, \ell<\beta_{1}<\alpha$, and $a_{1}$ increases from 0.6 for $r=2$ to 0.8 for $r=3$.

To investigate the effect of decay that increases with depth, contours of con- 
centration for discharged effluents from a point source at $\alpha=18$ on a short step seabed with $\ell=15$ are plotted in Figure 6 for $\sigma=1 / 2$ (decay rate linearly increases with depth) and for $\sigma=3 / 2$ (decay rate quadratically increases with depth). As shown in Figure 6 (left), the effect of decay with $\gamma=0.0003$ that increases with depth, is considerably small to be noticeable. Similar to that of discharging effluent plumes on the flat seabed (left of Figure 2), the shapes of the contours of concentration indicate that the presence of shoreline, that induces the effects of the no-slip condition, is still dominant.

The effect of a step increase in depth on dispersing discharged effluent plumes with $\gamma=0.0003$ and $\sigma=1 / 2$ is shown in Figure 6 (right) using the contour of $C=0.01$. If $r>3$, we observed that only small portions of the plumes entering and dispersing in the shallow region. The result suggests that the induced effect of the no-slip condition at $Y=0$ can be suppressed by the presence of a cross-stream sudden big drop in water depth.

Again, a plot of effluent flux is used to investigate the movement of discharged effluent plumes passes through the discontinuity line $Y=\ell$. In dimensionless form, the effluent flux on either side of the discontinuity line $Y=\ell$ are in the shallow region $0 \leq Y<\ell$, similar to that of the flat seabed, but with $\beta_{1}$ instead of $\alpha$ :

$$
F_{x}=b_{1} \sqrt{\frac{\lambda}{4 \pi X}}\left[\exp \left(-\frac{\lambda\left(Y-\beta_{1}\right)^{2}}{4 X}\right)+\exp \left(-\frac{\lambda\left(Y+\beta_{1}\right)^{2}}{4 X}\right)\right] \exp (-\gamma X)
$$

and

$$
\begin{aligned}
F_{y}= & b_{1} \sqrt{\frac{\lambda}{4 \pi X}}\left[\frac{\left(Y-\beta_{1}\right)}{2 X} \exp \left(-\frac{\lambda\left(Y-\beta_{1}\right)^{2}}{4 X}\right)\right. \\
& \left.+\frac{\left(Y+\beta_{1}\right)}{2 X} \exp \left(-\frac{\lambda\left(Y+\beta_{1}\right)^{2}}{4 X}\right)\right] \exp (-\gamma X) .
\end{aligned}
$$
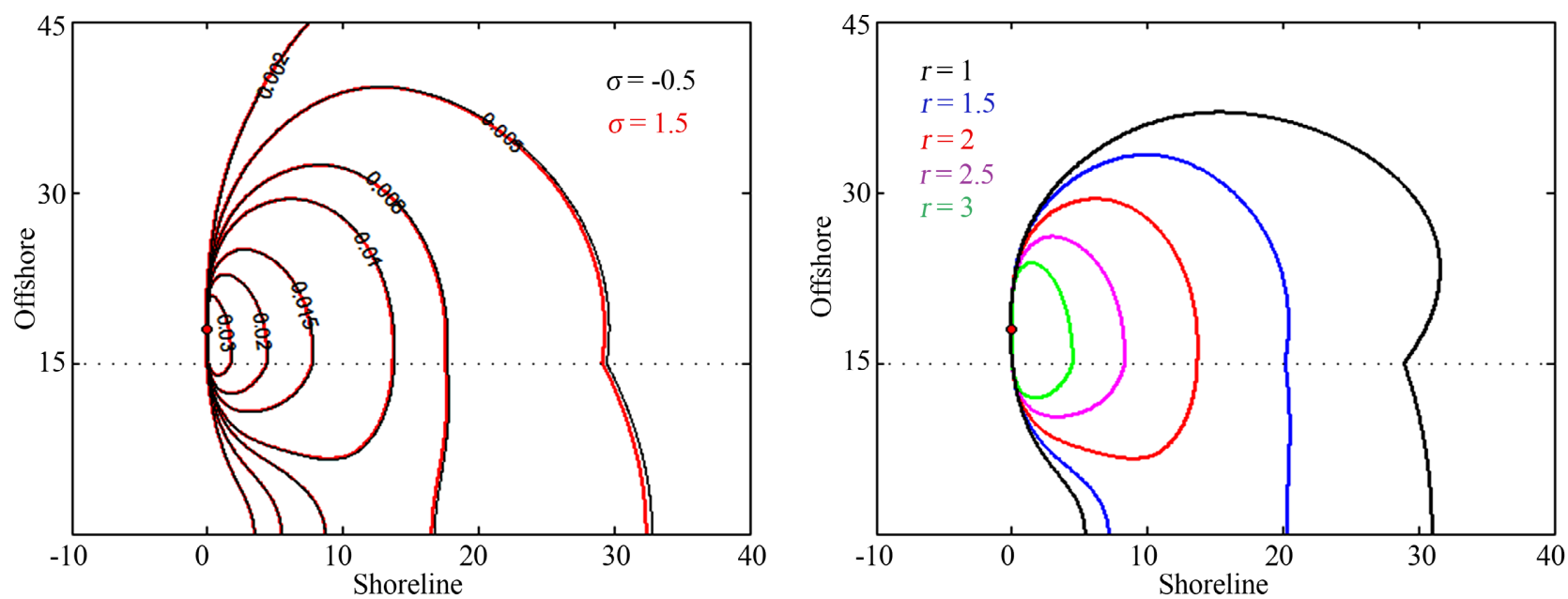

Figure 6. (left) Contours of concentration for discharging effluents with $\gamma=0.0003$ on a short step seabed with $\ell=15$ from a point source at $\alpha=18$ and (right) contours of $C=0.01$ and $\sigma=-1 / 2$. 
Within the deeper region $Y>\ell$, the flux in the $x$-direction is given by $\left(Q / h_{0}\right) F_{1 x}=h_{1} U_{1} c$ and in the $y$-direction is $\left(Q / h_{0}\right) F_{1 y}=-h_{1} D_{1} \partial c / \partial y$. In the dimensionless form,

$$
F_{1 x}=\sqrt{\frac{\lambda}{4 \pi r X}}\left[\exp \left(-\frac{\lambda(Y-\alpha)^{2}}{4 r X}\right)+a_{1} \exp \left(-\frac{\lambda(Y-2 \ell+\alpha)^{2}}{4 r X}\right)\right] \exp (-\gamma X)
$$

and

$$
\begin{aligned}
F_{1 y}= & \sqrt{\frac{\lambda}{4 \pi r X}}\left[\frac{(Y-\alpha)}{2 X} \exp \left(-\frac{\lambda(Y-\alpha)^{2}}{4 r X}\right)\right. \\
& \left.+a_{1} \frac{(Y-2 \ell+\alpha)}{2 X} \exp \left(-\frac{\lambda(Y-2 \ell+\alpha)^{2}}{4 r X}\right)\right] \exp (-\gamma X) .
\end{aligned}
$$

As shown in Figure 7, we observe that in the offshore region $Y \geq \alpha$, the effluent plume is tending to move further away. However, in the nearshore region $Y<\alpha$, the effluent plume spreads towards the discontinuity line $Y=\ell$, and it quickly enters the shallow region and reaches the shoreline at a relatively short downstream distance, then due to the no-slip condition, the plume continues to move in direction of the flow. It appears also that the effluent plume is trapped within the nearshore region $Y<\ell$.

The concentration levels at the discontinuity line $Y=\ell$ play an important role in determining the portions of discharged effluent plumes that crossing over and dispersing in the shallow region. By putting $Y=\ell$ in Equations (4) and (5), we obtain the concentration at the discontinuity line

$$
C_{0}(X, \ell)=C_{1}(X, \ell)=\frac{1+a_{1}}{2 r^{2}} \sqrt{\frac{\lambda}{\pi X}} \exp \left\{-\frac{\lambda(\alpha-\ell)^{2}}{4 r X}\right\} \exp \left(-\gamma r^{\sigma} X\right) .
$$

We note from Figure 8 that the position of maximum concentration occurs at
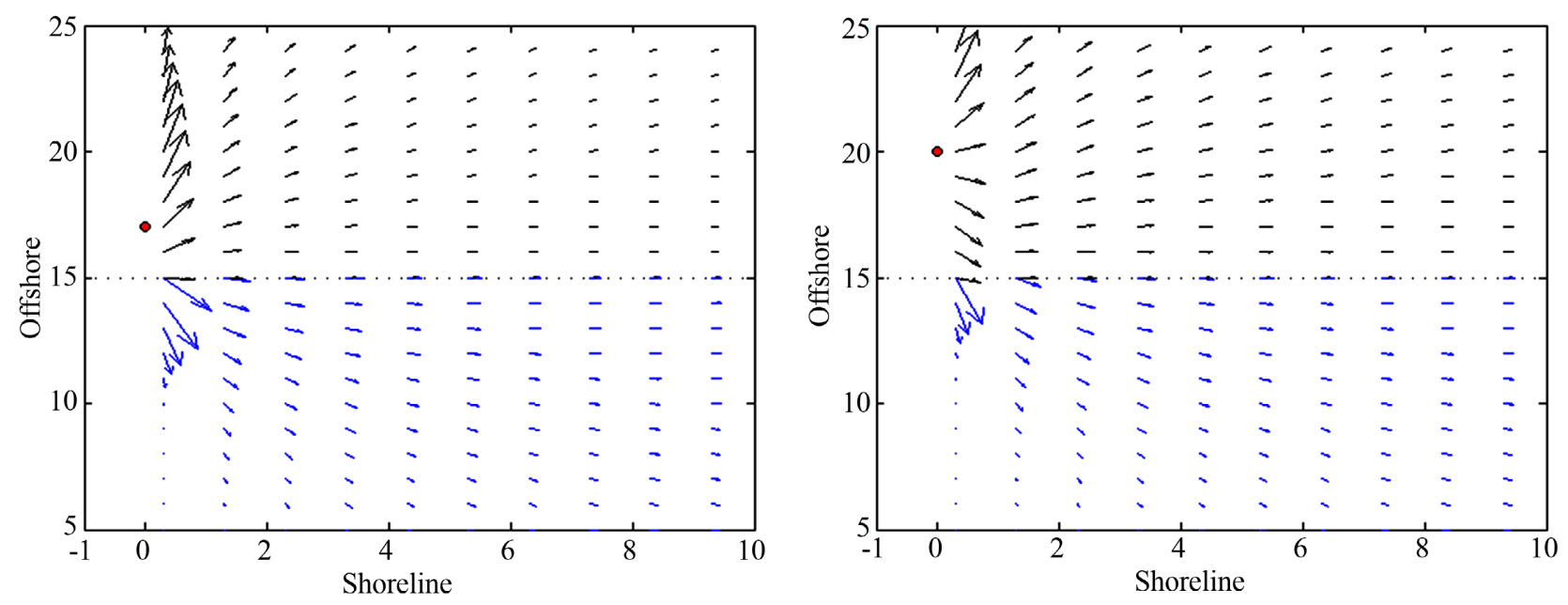

Figure 7. Flux of concentration for discharged effluents on a short step seabed with $\ell=15$ from a point source at $\alpha=17 \quad$ (left) and $\alpha=20$ (right) for the case of no decay $\gamma=0$. 


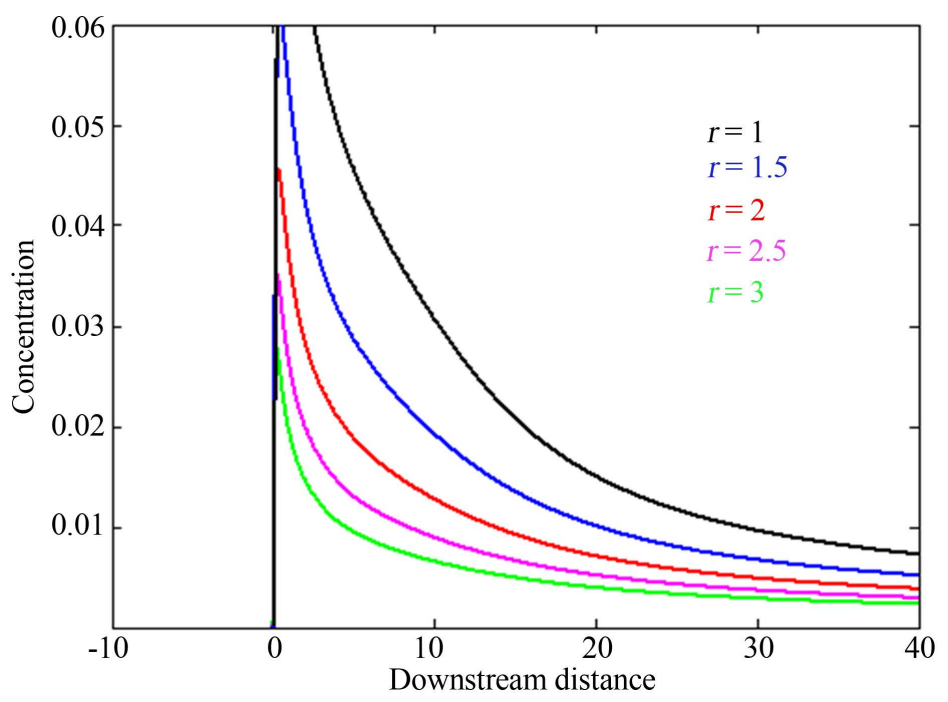

Figure 8. Concentration at the discontinuity line for discharging effluents with $\gamma=0.0003$ and $\sigma=1 / 2$ on a short step seabed with $\ell=15$ from a point source at $\alpha=18$.

a very short downstream distance, and thus, $a_{1}=\left(r^{2}-1\right) /\left(r^{2}+1\right)$. By differentiating, it has a maximum value

$$
C_{1 \max }=\frac{1}{r^{2}+1} \sqrt{\frac{1+\sqrt{1+4 \lambda \gamma r^{\sigma-1}(\alpha-\ell)^{2}}}{\pi(\alpha-\ell)^{2} / r}} \exp \left(-\frac{\sqrt{1+4 \lambda \gamma r^{\sigma-1}(\alpha-\ell)^{2}}}{2}\right),
$$

which occurs at $X_{1 \max }=\lambda(\alpha-\ell)^{2} / r\left[1+\sqrt{1+4 \lambda \gamma r^{\sigma-1}(\alpha-\ell)^{2}}\right]$. Note that the maximum value only depend on algebraic power of $(\alpha-\ell)$. For no decay $\gamma=0, \quad C_{1 \max }=\sqrt{2 r / \pi \mathrm{e}(\alpha-\ell)^{2}} /\left(r^{2}+1\right)$ and $X_{1 \max }=\lambda(\alpha-\ell)^{2} / 2 r$, which shows that $C_{1 \max }$ is inversely proportional to $r^{3 / 2}$, and the position of maximum concentration $X_{1 \max }$ is inversely proportional to $r$.

Due to small portions of discharged effluent plumes entering and dispersing in the shallow region, the maximum concentration that passes through the discontinuity line decreases from 0.0608 for $r=1.5$ to 0.0279 for $r=3$, a decrease of more than $54 \%$. In comparison with discharging effluents from a point source on the flat seabed, the maximum concentration at the shoreline is greatly reduced as a result of a step increase in depth at $\ell=15$. Finally, we note that as $\ell \rightarrow 0$, the concentration at the shoreline is

$$
C_{0}(X, 0)=\frac{1}{1+r^{2}} \sqrt{\frac{\lambda}{\pi X}} \exp \left(-\frac{\lambda \alpha^{2}}{4 r X}-\gamma r^{\sigma} X\right),
$$

which is $1 /\left(1+r^{2}\right)$ smaller than that of the flat seabed (see Equation (3)), and it has a maximum value given by

$$
C_{1 \max }=\frac{1}{1+r^{2}} \sqrt{\frac{1+\sqrt{1+4 \lambda \gamma r^{\sigma-1} \alpha^{2}}}{\pi \alpha^{2} / r}} \exp \left(-\frac{\sqrt{1+4 \lambda \gamma r^{\sigma-1} \alpha^{2}}}{2}\right) .
$$




\subsection{Long Step Seabed}

Sea outfalls should be sufficiently long to take the full benefit of stronger current and more depth to dilute discharged effluents. For simplicity, we assume that a source at $(X=0, Y=\alpha)$ is located sufficiently close to the line $Y=\ell$, where $70<\alpha \leq 80$ for discharging effluents in the deeper region and $60 \leq \alpha<70$ for discharging effluents in the shallow region, or else very small parts of the effluent plumes will be able to cross over the discontinuity line at $Y=\ell$. As previously mentioned, no imaginary source is needed to satisfy the boundary condition at $Y=0$.

\subsubsection{Discharging Effluents in the Deeper Offshore Region}

The solutions of the advection-diffusion equations are similar to that of discharging effluents on a short step seabed, but with different discharge rates of $a_{2}$ and $b_{2}$ instead of $a_{1}$ and $b_{1}$, respectively. For discharging effluents from a point source at $(X=0, Y=\alpha)$ where $\alpha>\ell$, the concentration $C_{0}(X, Y)=C_{0^{*}} \exp (-\gamma X)$ is obtained due to a virtual source at $\left(X=0, Y=\beta_{1}\right)$ discharging at a rate $b_{1}$ (see Equation (4)). Thus, in dimensionless form, the solution in the shallow region is

$$
C_{0}=b_{2} \sqrt{\frac{\lambda}{4 \pi X}} \exp \left\{-\frac{\lambda\left(Y-\beta_{1}\right)^{2}}{4 X}\right\} \exp (-\gamma X),
$$

and in the deeper region (see Equation (5)) is

$$
C_{1}=\frac{1}{2 r^{2}} \sqrt{\frac{\lambda}{\pi X}}\left[\exp \left\{-\frac{\lambda(Y-\alpha)^{2}}{4 r X}\right\}+a_{2} \exp \left\{-\frac{\lambda(Y-2 \ell+\alpha)^{2}}{4 r X}\right\}\right] \exp \left(-\gamma r^{\sigma} X\right) .
$$

The matching conditions at $Y=\ell$ are required for calculating $a_{2}, b_{2}$ and $\beta_{1}$, and thus, we obtain

$$
\beta_{1}=\ell+\frac{\alpha-\ell}{\sqrt{r}}, a_{2}=\frac{r^{2}-1}{r^{2}+1} \text { and } b_{2} \exp (-\gamma X)=\frac{2}{r^{2}+1} \exp \left(-\gamma r^{\sigma} X\right),
$$

and for example, the solution in the shallow region can be rewritten as

$$
C_{0}=\frac{1}{r^{2}+1} \sqrt{\frac{\lambda}{\pi X}} \exp \left\{-\frac{\lambda\left(Y-\beta_{1}\right)^{2}}{4 X}\right\} \exp \left(-\gamma r^{\sigma} X\right)
$$

which can be interpreted as the portions of discharged effluent plumes that cross over the line $Y=\ell$ and dispersing within the shallow nearshore region. We note that for $r>1, a_{2}<1, b_{2}>0$ and $\ell<\beta_{1}<\alpha$. It is also easy to verify that, for no decay $\gamma=0$ (and $\sigma=0$ ), then $a_{2}+b_{2}=1$; and if there is no depth change $r=1$ (and $\ell=0$ ), then $\beta_{1}=\alpha, a_{2}=0$ and $b_{2}=1$.

In comparison with discharging effluents on the flat seabed (right of Figure 2 ), it is clear that the symmetry about the centerline is broken due to the presence of a cross-stream sudden depth change at $\ell=70$, and most of the effluent plumes appear to be spreading mainly in the deeper region $Y>\ell$. As a result, 
the overall mixing on the step seabed is significantly enhanced.

The effect of variations in water depth on dispersing the discharged effluent plumes is shown in Figure 9 (right) using the contour of $C=0.0025$. If the deeper water is longer than three times that of the shallow $(r>3)$, we observed that only small portions of the plumes manage to enter and disperse in the shallow region $0 \leq Y<\ell$.

The concentration levels at the discontinuity line $Y=\ell$ play an important role in determining the portions of discharged effluent plumes that crossing over and dispersing in the shallow region. By putting $Y=\ell$, we obtain the concentration at the discontinuity line

$$
C_{0}(X, \ell)=C_{1}(X, \ell)=\frac{1}{r^{2}+1} \sqrt{\frac{\lambda}{\pi X}} \exp \left\{-\frac{\lambda(\alpha-\ell)^{2}}{4 r X}\right\} \exp \left(-\gamma r^{\sigma} X\right),
$$

which is exactly that of Equation (6), and it has a maximum value $C_{1 \max }$ that occurs at $X_{1 \max }$.

The concentration at the discontinuity line for discharging effluents from a point source at $\alpha=80$ on a long step seabed with $\ell=70$ is plotted in Figure 10 to show the effect of increasing $r$ for decay that is independent of depth $(\sigma=-1 / 2)$. The long tail of the graph is the result of the effluent plumes elongation in the downstream direction; and the maximum concentration value is unaffected by increasing value of $\sigma$. Due to small portions of discharged effluent plumes entering and dispersing in the shallow region, the maximum concentration that passes through the discontinuity line decreases from 0.0084 for $r=3$ to 0.0042 for $r=5$, a decrease of about $50 \%$. We conclude that, in comparison to the discharged effluents from a point source at $\alpha=80$ on the flat seabed, the maximum concentration at the shoreline is greatly reduced as a result of a step increase in depth at $\ell=70$.
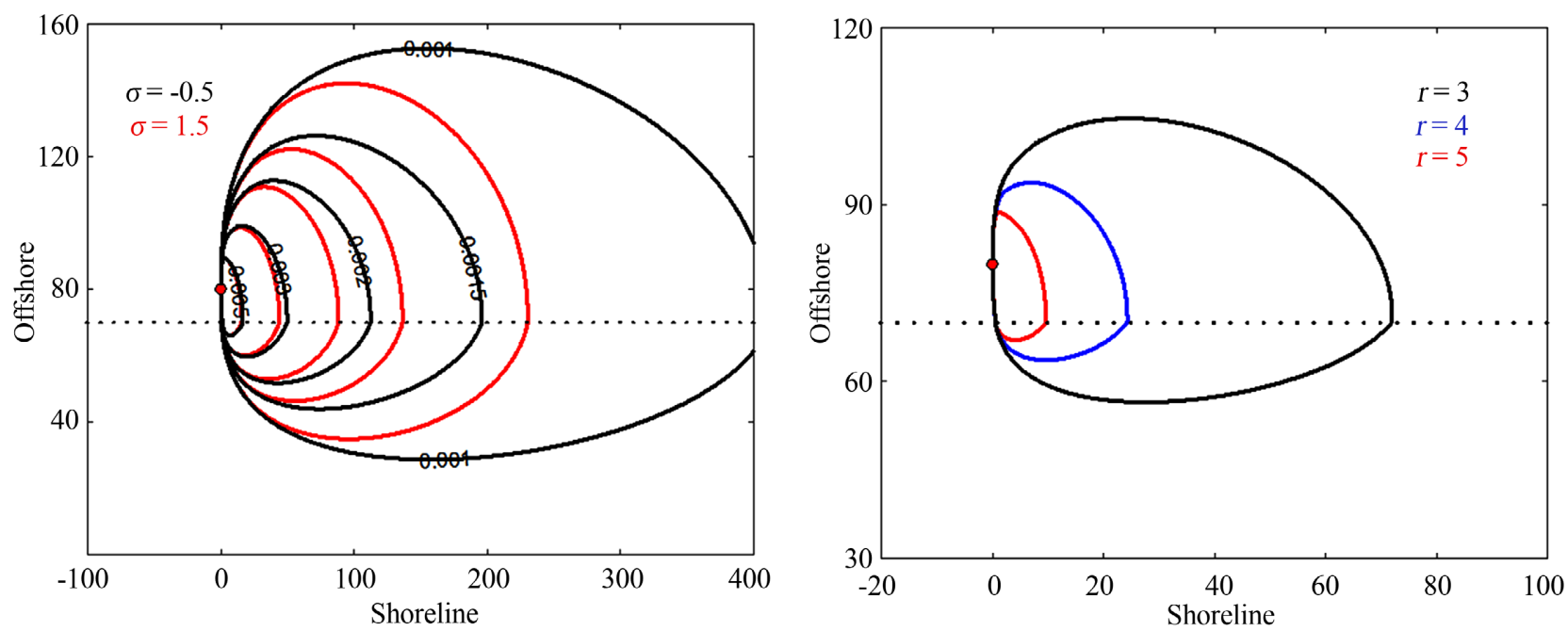

Figure 9. (left) Contours of concentration for discharging effluents with $\gamma=0.0003$ on a long step seabed with $\ell=70$ and $r=3$ from a point source at $\alpha=80$; and (right) contours of $C=0.0025$ and $\sigma=-1 / 2$. 


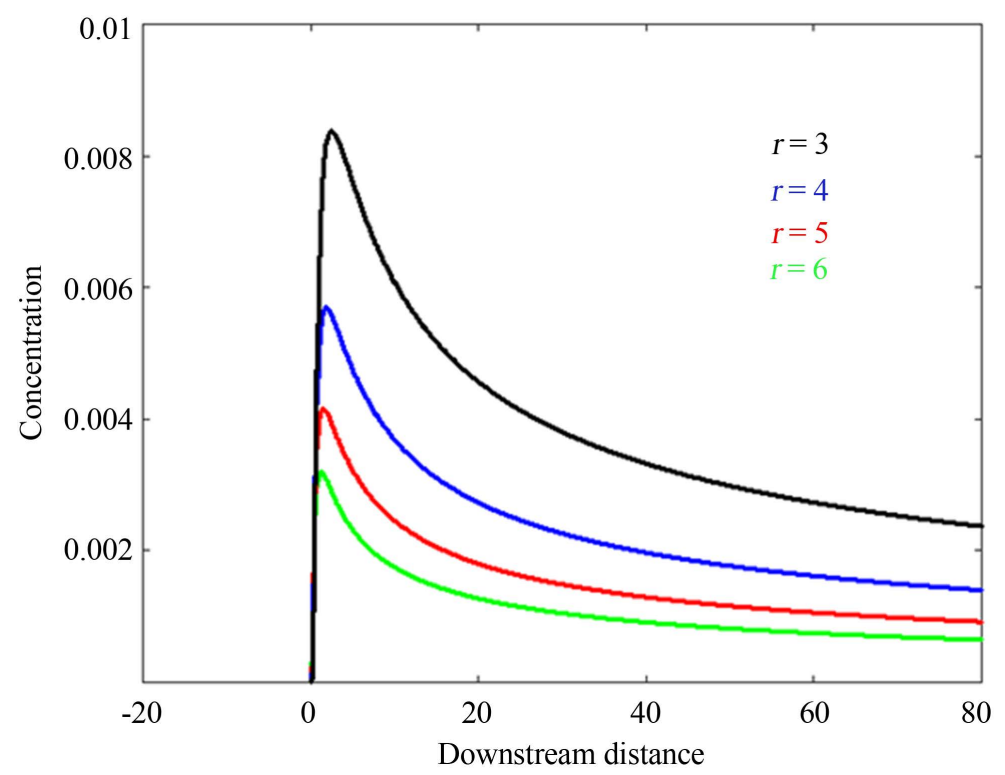

Figure 10. Concentration at the discontinuity line for discharging effluents with $\gamma=0.0003$ and $\sigma=-1 / 2$ on a long step seabed with $\ell=70$ from a point source at $\alpha=80$.

\subsubsection{Discharging Effluents in the Shallow Nearshore Region}

For discharging effluents from a point source at $(X=0, Y=\alpha)$, where $\alpha<\ell$, the concentration $C_{1}(X, Y)$ is obtained due to a virtual point source at $\left(X=0, Y=\beta_{2}\right)$ discharging at a rate $b_{3}$. Thus, in dimensionless form, the advection-diffusion equation for $C_{1^{*}}(X, Y)$ is given by

$$
\frac{\partial C_{1^{*}}}{\partial X}-\frac{r}{\lambda} \frac{\partial^{2} C_{1^{*}}}{\partial Y^{2}}=\frac{b_{3}}{r^{3 / 2}} \delta(X) \delta\left(Y-\beta_{2}\right),
$$

and the solution for $X \geq 0$ is

$$
C_{1}=\frac{b_{3}}{2 r^{2}} \sqrt{\frac{\lambda}{\pi X}} \exp \left\{-\frac{\lambda\left(Y-\beta_{2}\right)^{2}}{4 r X}\right\} \exp \left(-\gamma r^{\sigma} X\right) .
$$

The concentration $C_{0}(X, Y)$ is obtained due to a point source at $(X=0, Y=\alpha)$ and an imaginary source at $(X=0, Y=2 \ell-\alpha)$ discharging at a different rate $a_{3}$. The advection-diffusion equation for $C_{0^{*}}(X, Y)$ is given by

$$
\frac{\partial C_{0^{*}}}{\partial X}-\frac{1}{\lambda} \frac{\partial^{2} C_{0^{*}}}{\partial Y^{2}}=\delta(X)\left[\delta(Y-\alpha)+a_{3} \delta(Y-2 \ell+\alpha)\right],
$$

and the solution for $X \geq 0$ is

$$
C_{0}=\sqrt{\frac{\lambda}{4 \pi X}}\left[\exp \left\{-\frac{\lambda(Y-\alpha)^{2}}{4 X}\right\}+a_{3} \exp \left\{-\frac{\lambda(Y-2 \ell+\alpha)^{2}}{4 X}\right\}\right] \exp (-\gamma X) .
$$

From the matching conditions at $Y=\ell$ we obtain

$$
\beta_{2}=\ell-\sqrt{r}(\ell-\alpha), \quad a_{3}=\frac{1-r^{2}}{1+r^{2}} \text { and } \frac{b_{3}}{r^{2}} \exp \left(-\gamma r^{\sigma} X\right)=\frac{2}{1+r^{2}} \exp (-\gamma X) \text {, }
$$

and thus, Equation (8) can be rewritten as 


$$
C_{1}=\frac{1}{1+r^{2}} \sqrt{\frac{\lambda}{\pi X}} \exp \left\{-\frac{\lambda\left(Y-\beta_{2}\right)^{2}}{4 r X}\right\} \exp (-\gamma X),
$$

which is independent of $\sigma$, i.e. there are no effects of variability of decay with depth, and it can be interpreted as the portions of discharged effluent plumes that escaping out to the deeper offshore region. We noted that, for no decay $\gamma=0$, then $a_{3}+b_{3}=1$; and if there is no depth change $r=1$ (and $\ell=0$ ), then $\beta_{2}=\alpha, a_{3}=0$ and $b_{3}=1$. Also for $r>1, a_{3}<0, b_{3}<1$ and $\alpha<\beta_{2}<\ell$.

As plotted in Figure 11 (left), it is clear that from the contours of concentration, due to the presence of a sudden depth change at $\ell=70$, small portions of the effluent plumes escaping into the deeper region $Y>\ell$ and most of the plumes are remaining and dispersing in the shallow region $0 \leq Y<\ell$. Using the contour of $C=0.005$, the effects of a step increase in depth are shown in Figure 11 (right). If $r>3$, we observed that no discharged effluent plumes escaping into the deeper region and the entire plumes are dispersed in the shallow region.

Next, by putting $Y=\ell$, we obtain the concentration at the discontinuity line

$$
C_{0}(X, \ell)=C_{1}(X, \ell)=\frac{1}{r^{2}+1} \sqrt{\frac{\lambda}{\pi X}}\left[\exp \left\{-\frac{\lambda(\ell-\alpha)^{2}}{4 X}\right\}\right] \exp (-\gamma X) \text {. }
$$

By differentiating, the maximum value is

$$
C_{2 \max }=\frac{1}{r^{2}+1} \sqrt{\frac{1+\sqrt{1+4 \lambda \gamma(\ell-\alpha)^{2}}}{\pi(\ell-\alpha)^{2}}} \exp \left(-\frac{\sqrt{1+4 \lambda \gamma(\ell-\alpha)^{2}}}{2}\right),
$$

which occurs at $X_{2 \max }=\lambda(\ell-\alpha)^{2} /\left[1+\sqrt{1+4 \lambda \gamma(\ell-\alpha)^{2}}\right]$. Again, the maximum value only depend on algebraic power of $(\ell-\alpha)$. For no decay $\gamma=0$, $C_{2 \max }=\sqrt{2 / \pi \mathrm{e}(\ell-\alpha)^{2}} /\left(r^{2}+1\right)$ and $X_{2 \max }=\lambda(\ell-\alpha)^{2} / 2$. This shows also that
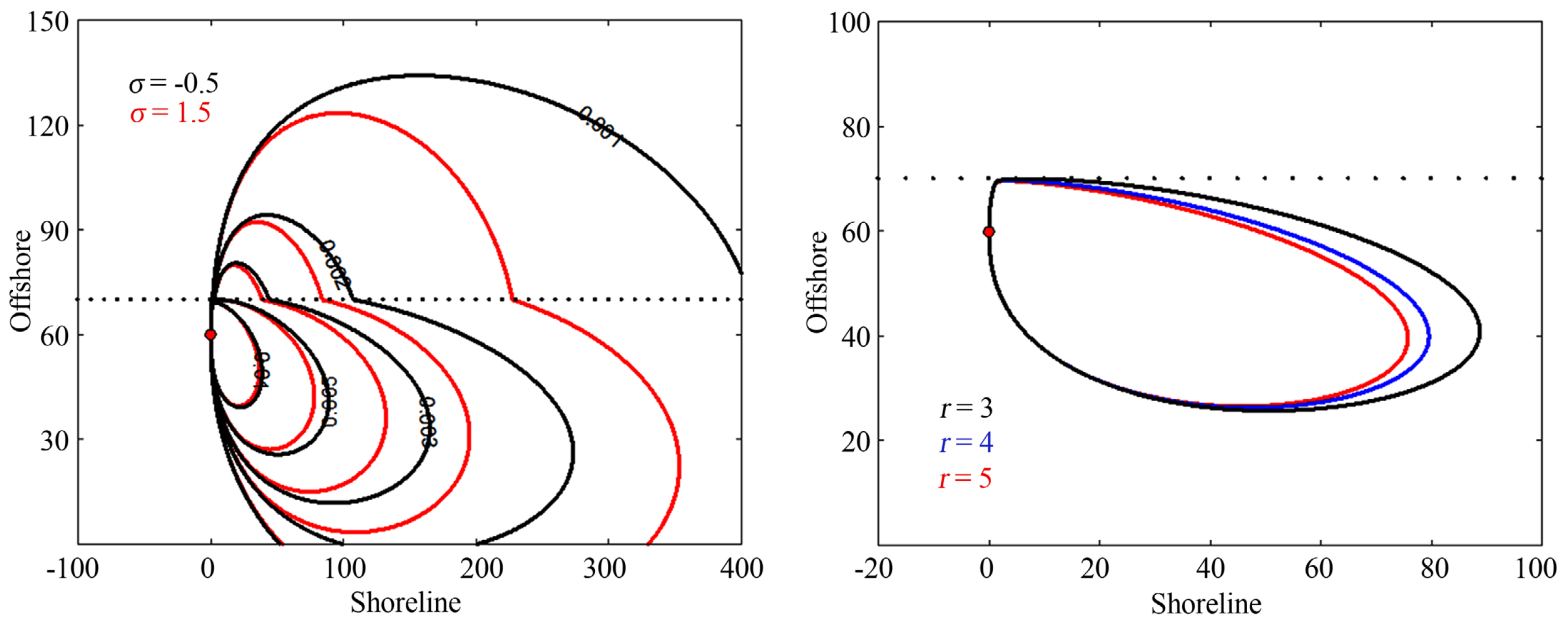

Figure 11. (left) Contours of concentration for discharging effluents with $\gamma=0.0003$ on a long step seabed with $\ell=70$ and $r=3$ from a point source at $\alpha=60$ and (right) contours of $C=0.005$ and $\sigma=-1 / 2$. 


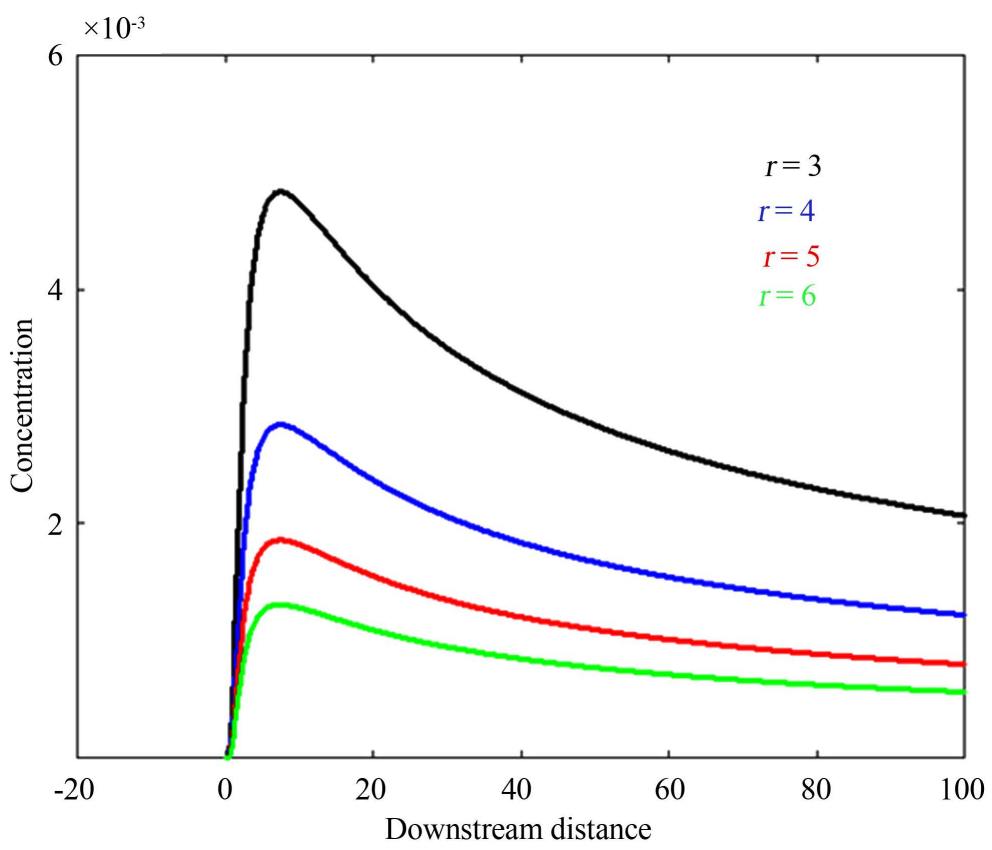

Figure 12. Concentration at the discontinuity line for discharging effluents with $\gamma=0.0003$ and $\sigma=-1 / 2$ on a long step seabed with $\ell=70$ from a point source at $\alpha=60$.

$C_{2 \max }$ is inversely and quadratically proportional to $r$, however, the position of maximum concentration $X_{2 \max }$ is independent of $r$, as shown in Figure 12.

As shown in Figure 12, due to small portions of the discharged effluent plumes escaping to the deeper region, the maximum concentration that passes through the discontinuity line decreases from 0.0048 for $r=3$ to 0.0019 for $r=5$, a decrease of about $60 \%$. We conclude that, in comparison to discharging effluents from a point source at $\alpha=60$ on a flat seabed, the maximum concentration at the shoreline is reduced as a result of a step increase in depth at $\ell=70$. We note that as $\ell \rightarrow 0$, the concentration at the shoreline is exactly that of Equation (7),

$$
C_{0}(X, 0)=\frac{1}{1+r^{2}} \sqrt{\frac{\lambda}{\pi X}} \exp \left(-\frac{\lambda \alpha^{2}}{4 X}-\gamma X\right) .
$$

\section{Concluding Remarks}

Mathematical models are presented using a two-dimensional advection-diffusion equation with a point source to study the effects of a sudden water depth change and decay in mixing and dispersion of steady discharged effluents through a sea outfall in coastal waters. Due to the small nature of decay rates, for shorter sea outfalls, the results show that it is difficult to overcome the slow mixing processes in the nearshore region due to the no-slip condition at the shoreline. A large increase in water depth for more than three times that of the shallow region is needed to suppress the effect of the no-slip condition.

For steady discharges from a point source in the deeper region, the results 
show that in comparison to that of a flat seabed, the maximum concentration at the shoreline can be greatly reduced by the presence of a step increase in water depth. However, for steady discharges in the shallow region, the reduction is inversely proportional to the quadratic of depth ratio.

The model extensions to account for multiple point source discharging effluents on a long step seabed have been published [14] [15], and for steady effluent discharges on a uniformly sloping seabed will be presented in a subsequent paper.

\section{Conflicts of Interest}

The authors declare no conflicts of interest regarding the publication of this paper.

\section{References}

[1] Gould, D.J. and Munro, D. (1981) Relevance of Microbial Mortality to Outfall Design. In: Institute of Civil Engineers, Coastal Discharges. Engineering Aspect and Experience, Thomas Telford, Ltd., London, 45-50.

[2] Institution of Civil Engineers (1989) Long Sea Outfalls. Thomas Telford, Ltd., London.

[3] Roberts, P.J.W., Salas, H.J., Reef, F.M., Libhaber, M., Labe, A. and Thomson, J.C. (2010) Marine Wastewater Outfalls and Treatment Systems. Vol. 9, International Water Association (IWA) Publishing, London. https://doi.org/10.2166/9781780401669

[4] Macqueen, J.F. and Preston, R.W. (1983) Cooling Water Discharges into a Sea with a Sloping Bed. Water Research, 17, 389-395.

https://doi.org/10.1016/0043-1354(83)90134-3

[5] Purnama, A. and Al-Barwani, H.H. (2006) Spreading of Brine Waste Discharges into the Gulf of Oman. Desalination, 195, 26-31.

https://doi.org/10.1016/j.desal.2005.09.036

[6] Roberts, D.A., Johnston, E.L. and Knott, N.A. (2010) Impacts of Desalination Plants Discharges on the Marine Environment: A Critical Review of Published Studies. Water Research, 44, 5117-5128. https://doi.org/10.1016/j.watres.2010.04.036

[7] Lattemann, S. and Hopner, T. (2008) Environmental Impact and Impact Assessment of Seawater Desalination. Desalination, 220, 1-15.

https://doi.org/10.1016/j.desal.2007.03.009

[8] Ahmad, N. and Baddour, R.E. (2014) A Review of Sources, Effects, Disposal Methods, and Regulations of Brine into Marine Environments. Ocean and Coastal Management, 87, 1-7. https://doi.org/10.1016/j.ocecoaman.2013.10.020

[9] Mebine, P. and Smith, R. (2006) Effects of Contaminant Decay on the Diffusion Centre of a River. Environmental Fluid Mechanics, 6, 101-114. https://doi.org/10.1007/s10652-006-0004-2

[10] Mebine, P. and Smith, R. (2009) Effect of Pollutant Decay on Steady-State Concentration Distributions in Variable Depth Flow. Environmental Fluid Mechanics, 9, Article No. 573. https://doi.org/10.1007/s10652-009-9156-1

[11] Smith, R. (1981) Effects of Non-Uniform Currents and Depth Variations upon Steady Discharges in Shallow Water. Journal of Fluid Mechanics, 110, 373-380. https://doi.org/10.1017/S0022112081000797 
[12] Kay, A. (1987) The Effect of Cross-Stream Depth Variations upon Contaminant Dispersion in a Vertically Well-Mixed Current. Estuarine, Coastal Shelf Science, 24, 177-204. https://doi.org/10.1016/0272-7714(87)90064-3

[13] Purnama, A., Al Maamari, H.A. and Balakrishnan, E. (2017) Optimal Outfall Systems for Nearshore Effluent Discharges on Eroded Sandy Beaches. Journal of Coupled Systems and Multiscale Dynamics, 5, 217-224.

https://doi.org/10.1166/jcsmd.2017.1134

[14] Purnama, A., Al-Maamari, H.A. and Balakrishnan, E. (2019) Decay Model for Dispersion of Coastal Discharged Effluents from Multiport Diffusers in the Far-Field. International Journal of New Innovations in Engineering and Technology, 12, 8-18.

[15] Purnama, A., Al-Maamari, H.A, Al-Muqbali, A.A. and Balakrishnan, E. (2020) The Effect of a Step Change in Seabed Depth on Spreading Discharged Brine Effluents from a Two-Outfall System. Journal of Mathematical and Computational Science, 10, 758-777.

[16] Chin, D.A. and Roberts, P.J.W. (1985) Model of Dispersion in Coastal Waters. Journal of Hydraulic Engineering, 111, 12-28. https://doi.org/10.1061/(ASCE)0733-9429(1985)111:1(12)

[17] Wood, I.R. (1993) Asymptotic Solutions and Behavior of Outfall Plumes. Journal of Hydraulic Engineering, 119, 553-580. https://doi.org/10.1061/(ASCE)0733-9429(1993)119:5(553)

[18] Li, C.H., Pan, X.S., Ke, J. and Dong, X.T. (2015) Comparison of 2D and 3D Models of Salinity Numerical Simulation. Polish Maritime Research, 22, 26-29. https://doi.org/10.1515/pomr-2015-0028

[19] Craig, D.L., Fallowfield, H.J. and Cromar, N.J. (2002) Comparison of Decay Rates of Faecal Indicator Organisms in Recreational Coastal Water and Sediment. Water Supply, 2, 131-138. https://doi.org/10.2166/ws.2002.0095

[20] Adcroft, A., Hallberg, R., Dunne, J.P., Samuels, B.L. and Galt, J.A. (2010) Simulations of Underwater Plumes of Dissolved Oil in the Gulf of Mexico. Geophysical Research Letters, 37, Article ID: L18605 (5 pages). https://doi.org/10.1029/2010GL044689

[21] Shukla, V.P. (2002) Analytical Solutions for Unsteady Transport Dispersion of Nonconservative Pollutant with Time-Dependent Periodic Waste Discharge Concentration. Journal of Hydraulic Engineering, 128, 866-869. https://doi.org/10.1061/(ASCE)0733-9429(2002)128:9(866)

[22] Ostendorf, D.W. (1982) Longshore Dispersion over a Flat Beach. Journal of Geophysical Research, 87, 4241-4248. https://doi.org/10.1029/JC087iC06p04241 